\title{
Microwave-assisted sequential one-pot protocol to benzothiadiazin-3-one-1,1-dioxides via a copper-catalyzed $\mathrm{N}$ - arylation strategy
}

\author{
Alan Rolfe and Paul R. Hanson* \\ Department of Chemistry, University of Kansas, 1251 Wescoe Hall Drive, Lawrence, KS \\ 66045-7582, USA \\ KU Center of Excellence in Chemical Methodologies and Library Development, University of \\ Kansas, 1501 Wakarusa Drive, Lawrence, KS 66047, USA
}

\begin{abstract}
A microwave-assisted, sequential, one-pot protocol has been developed for the synthesis of a variety of benzothiadiazin-3-one-1,1-dioxides. This protocol utilizes a copper-catalyzed $\mathrm{N}$-arylation of $\alpha$ bromo-benzenesulfonamides with a number of amines to generate the corresponding 2aminobenzenesulfonamides, which undergo cyclization to the desired sultams using carbonyl diimidazole (CDI). A range of conditions was evaluated for the key $\mathrm{C}-\mathrm{N}$ bond formation step with tolerance toward functionalized amines.
\end{abstract}

\section{Introduction}

The development of protocols for the synthesis of skeletally diverse heterocyclic scaffolds is a critical step in the drug discovery process. The growing demand for libraries of small molecules as potential small molecule therapeutic agents for high-throughput screening presents challenging opportunities in this field. One-pot strategies are highly efficient pathways to rapidly synthesize complex heterocyclic molecules from simple substrates. ${ }^{1}$ When coupled with transition metal-catalyzed processes, one-pot processes enable the generation of complex heterocyclic scaffolds from simple building blocks. In this regard, $\alpha$-haloarylsulfonamides represent an attractive building block for the production of benzofused sultams. ${ }^{2,3}$

Sultams and their sulfonamide precursors possess a number of advantageous chemical properties making them ideal building blocks for the titled process, the most prominent of these include: (i) click coupling between starting $\alpha$-halobenzenesulfonyl chlorides and amines under mild conditions, (ii) the $\alpha$-halo group can be utilized in transition metal-catalyzed cross coupling (iii) the $\alpha$-halo group enhances the acidity of the aryl sulfonamide $\mathrm{N}-\mathrm{H}$ enabling Mitsunobu and conventional alkylation reactions to occur under mild conditions, and (iv) the commercial availability of a variety of substituted $\alpha$-halo benzenesulfonyl chlorides. Taken collectively, these attributes have guided our efforts to develop a microwave-assisted, sequential one-pot protocol for the synthesis of benzothiazdiazin-3-one-1,1-dioxides based on a pivotal copper-catalyzed $\mathrm{N}$-arylation strategy.

Traditionally, sultams have been synthesized using a number of classical cyclization protocols such as Friedel-Crafts, [3+2] cycloadditions, Diels-Alder reactions, and recently the 
application of oxa- and aza-Michael reactions. ${ }^{4}$ Notably, there have been a number of transition metal-catalyzed protocols reported for the generation of diverse sultams.3, 5

In addition to their inherent chemical properties, sultams have emerged as important targets for drug discovery due to their potent biological activities. In particular, benzothiadiazin-3one-1,1-dioxides and their derivatives have shown promising activity, including hypoglycemic, ${ }^{6}$ anti-HIV,${ }^{7}$ RSV inhibitory activity, ${ }^{8}$ as well asand serving as selective antagonists of CXR2 (Fig. 1). ${ }^{9}$

\section{Results and discussion}

Since the observation of copper-catalyzed coupling of a arylbromide with an acetanilide by Goldberg in $1907,{ }^{10}$ copper-catalyzed $\mathrm{N}$-arylation represents an effective reaction for the formation of $\mathrm{C}-\mathrm{N}$ and $\mathrm{C}-\mathrm{O}$ bonds. ${ }^{11}$ Early reports classically required harsh reaction conditions and stoichiometric quantities of copper. Seminal work by Buchwald, Hartwig and Ley reported notable advances in both ligands and reduced reaction temperatures for coppercatalyzed couplings. ${ }^{12}$

Traditionally, benzothiadiazin-3-one-1,1-dioxides have been synthesized in a number of linear protocols. ${ }^{6-9,13}$ Envisioning a copper-catalyzed approach to benzothiadiazin-3-one-1,1dioxides, a variety of conditions were evaluated to probe and subsequently optimize the Narylation of allyl amine with $\mathrm{N}$-allyl-2-bromo-4-fluorobenzenesulfonamide $\mathbf{1}$ to yield $\mathrm{N}$ allyl-2-(allylamino)-4-fluorobenzenesulfonamide 2 (Scheme 1, Table 1). ${ }^{14}$ An array of copper sources (Table 1, entries 1-3) and ligands (Table 1, entries 4-7) were initially evaluated followed by a survey of reaction solvent. Under conventional heating, the desired sulfonamide 2 , could be isolated in $92 \%$ yield after $6 \mathrm{~h}$.

Further optimization was achieved using microwave irradiation, which reduced reaction times to $11 \mathrm{~min}$ at $150{ }^{\circ} \mathrm{C}$ with comparable yields (Table 1 , entry 8 vs 11 ). ${ }^{15}$ With these results in hand, a number of 2-aminobenzenesulfonamide derivatives were synthesized to demonstrate the versatility of the protocol with a variety of amines, amides, and sulfonamide starting materials (Scheme 2, Table 2).

With an array of 2-aminobenzenesulonfamides in hand, cyclization to the corresponding benzothiadiazin-3-one-1,1-dioxides with carbonyl diimidazole (CDI) was achieved in excellent yields under thermal conditions (Scheme 3, Table 3). ${ }^{13 \mathrm{~b}, 17}$

Finally with both protocols in hand, a sequential, two-step, one-pot approach was achieved whereby microwave irradiation afforded the desired benzothiadiazin-3-one-1,1-dioxides in good yield (Scheme 4). ${ }^{18}$ To achieve this, the CDI cyclization was conducted under microwave irradiation following the initial copper-catalyzed step in the same microwave vial. This required a change of solvent to DMF which was the optimum compatible solvent for both the $\mathrm{N}$-arylation and CDI cyclization steps while maintaining good yields.

In conclusion, we have developed a microwave-assisted, copper-catalyzed, sequential, onepot synthesis of benzothiadiazin-3-one-1,1-dioxides. A variety of derivatives of benzothiadiazin-3-one-1,1-dioxides can be rapidly accessed by combining a copper-mediated $\mathrm{N}$-arylation followed by cyclization with CDI. Further efforts toward employment of this method in library production will be published in due course.

\section{Supplementary Material}

Refer to Web version on PubMed Central for supplementary material. 


\section{Acknowledgments}

This publication was made possible by the Pilot-Scale Libraries Program (P41 GM076302), the National Institutes of General Medical Sciences (KU Chemical Methodologies and Library Development Center of Excellence P50 GM069663) and by Grant Number P20 RR015563 from the National Center for Research Resources, a component of the National Institutes of Health, and the State of Kansas. Its contents are solely the responsibility of the authors and do not necessarily represent the official view of the NCRR or NIH.

\section{References and notes}

1. (a) Fustero S, Jiméez D, Sánchez-Roselló M, del Pozo C. J. Am. Chem. Soc 2007;129:6700-6701. [PubMed: 17480078] (b) Bi H-P, Liu X-Y, Gou F-R, Guo L-N, Duan X-H, Shu X-Z, Liang Y-M. Angew. Chem., Int. Ed 2007;46:7068-7071. (c) Zeng Y, Reddy DS, Hirt E, Aubé J. Org. Lett 2004;6:4993-4995. [PubMed: 15606118] (d) Kirschbaum S, Waldmann H. Tetrahedron Lett 1997;38:2829-2832.

2. For the use of a-halo arylsulfonamides in synthesis of sultams see: (a) Grigg R, York M. Tetrahedron Lett 2000;41:7255-7258. (b) Evans P, McCabe T, Morgan BS, Reau S. Org. Lett 2005;7:44-46. (c) Vasudevan A, Tseng P-S, Djuric SW. Tetrahedron Lett 2006;47:8591-8593. (d) Paquette LA, Dura R, Fosnaugh N, Stephanian MJ. Org. Chem 2006;71:8445-8483. For radical cyclization: (f) Bressy C, Menant C, Piva O. Synlett 2005:577-582. For alkyne 6-endo cyclizations: (g) Barange DK, Nishad TC, Swamy K, Bandameedi V, Kumar D, Bukkapattanam RS, Vyas K, Pal MJ. Org. Chem 2007;72:8547-8550.

3. (a) Zhou A, Hanson PR. Org. Lett 2008;10:2951-2954. [PubMed: 18553974] (b) Jeon KO, Rayabarapu D, Rolfe A, Volp K, Omar I, Hanson PR. Tetrahedron 2009:4992-5000. [PubMed: 20161277]

4. (a) Zhou A, Rayabarapu KD, Hanson PR. Org. Lett 2009:531-534. [PubMed: 19115841] (b) Rolfe A, Young K, Hanson PR. Eur. J. Org. Chem 2008:5254-5262. (c) Rolfe A, Young K, Volp KA, Schoenen F, Neuenswander B, Lushington GH, Hanson PR. J. Comb. Chem 2009;11:732-738. [PubMed: 19505109] (d) Rayabarapu DK, Zhou A, Jeon KO, Samarakoon T, Rolfe A, Siddiqui H, Hanson PR. Tetrahedron 2009;65:3180-3188. and references cited therein. [PubMed: 20161276]

5. (a) McReynolds MD, Dougherty JM, Hanson PR. Chem. Rev 2004;104:2239-2258. [PubMed: 15137790] (b) Jiménez-Hopkins M, Hanson PR. Org. Lett 2008;10:2951-2954. and references cited therein. [PubMed: 18553974]

6. Wales JK, Krees SV, Grant AM, Vikroa JK, Wolff F, Pharm WJ. Exp. Ther 1968;164:421-432.

7. (a) Buckheit WR, Fliaka-Boltz V, Decker DW, Roberson LJ, Pyle CA, White LE, Bowden BJ, McMahon JB, Boyd MR, Bader JP, Nickell DG, Barth H, Antonucci TK. Antiviral Res 1994;25:4356. [PubMed: 7529014] (b) Arranz EM, Diaz JA, Ingate ST, Witvrouw M, Pannecouque C, Balzarini J, Clercq ED, Vega S. Bioorg. Med. Chem 1999;7:2811-2822. [PubMed: 10658585]

8. Combrink KD, Gulgeze HB, Thuring JW, Yu K-L, Civiello RL, Zhang Y, Pearce BC, Yin Z, Langley DR, Kadow KF, Cianci CW, Li Z, Clarke J, Genovesi EV, Medina I, Lamb L, Yang Z, Zadjura L, Krystal M, Meanwell NA. Bioorg. Med. Chem. Lett 2007;17:4784-4790. [PubMed: 17616396]

9. Wang Y, Busch-Petersen J, Wang F, Ma L, Fu W, Kerns JK, Jin J, Palovich MR, Shen J-K, Burman M, Foley JJ, Schmidt DB, Hunsberger GE, Sarau HM, Widdowson KL. Bioorg. Med. Chem. Lett 2007;17:3864-3867. [PubMed: 17524641]

10. Golberg I. Ber. Dtsch. Chem. Ges 1907;40:4541.

11. (a) George TG, Endeshaw MM, Morgan RE, Mahasenan KV, Delfin DA, Mukherjee MS, Yakovich AJ, Fotie J, Li C, Werbovetz KA. Bioorg. Med. Chem. Lett 2007;15:6071-6079. (b) Zhu L, Li G, Luo L, Guo P, Lan J, You J. J. Org. Chem 2009;74:2200-2202. [PubMed: 19196026] (c) Feng E, Huang H, Zhou Y, Ye D, Jiang H, Liu H. J. Org. Chem 2009;74:2846-2849. [PubMed: 19256470] (d) Stieter ER, Bhayana B, Buchwald SL. J. Am. Chem. Soc 2009;31:78-88.

12. (a) Klapars A, Abtilla JC, Huang X, Buchwald SL. J. Am. Chem. Soc 2001;123:7727. [PubMed: 11481007] (b) Hartwig JF. Synlett 2006:1283. (c) Ley SV, Thomas AW. Angew. Chem. Int. Ed 2003;42:5400.

13. (a) Boverie S, Antoine M-H, Somers F, Becker B, Sebille S, Ouedraogo R, Counerotte S, Pirotte B, Lebrun P, Tullio P. J. Med. Chem 2005;48:3492-3503. [PubMed: 15887958] (b) Khazi IA, Jung YS. Lett. Org. Chem 2007;4:423-428. 
14. (a) Lai G, Gum RJ, Daly M, Fry EH, Hutchins C, Abad-Zapatero C, von Geldern TW. Bioorg. Med. Chem. Lett 2006;16:1807-1810. [PubMed: 16446092] (b) Freeman HS, Butler JR, Freedman LD. J. Org. Chem 1978;43:4975-4978. (c) Bacon RGR, Rennison SG. J. Chem. Soc 1969:312. (d) Wu YJ, He H, L'Heureux A. Tetrahedron Lett 2004;44:4217-4218. (e) Kim JK, Lee Y, Lee J, Do Y, Chang S. J. Org. Chem 2008;73:9454-9457. [PubMed: 18956843]

15. While final yields obtained with DMSO, using DMF gave results within 5\% experimental error. However it was found that under microwave conditions at $150{ }^{\circ} \mathrm{C}$, a small amount of by-product was formed from the addition of dimethylamine into the 4-F position of the benzene ring in a $\mathrm{S}_{\mathrm{N}} \mathrm{Ar}$ mechanism. It is proposed that a small amount of dimethylamine is produced from the decomposition of DMF under these conditions and hence DMSO is a better solvent for such substrates.

16. General procedure for the $N$-arylation of $\alpha$-bromobenzenesulfonamides: Into a microwave reaction vial was added sulfonamide ( $0.17 \mathrm{mmol}, 1$ equiv), $\mathrm{CuI}$ ( $0.017 \mathrm{mmol}$. 0.1 equiv), 1,10-phenanthroline (0.034 mmol, 0.2 equiv), $\mathrm{Cs}_{2} \mathrm{CO}_{3}$ (0.34 mmol, 2 equiv), dry DMSO or DMF (0.5 M), and amine (0.2 mmol, 1.2 equiv). The reaction was heated in the microwave (Biotage initiator, www.biotage.com) at $150{ }^{\circ} \mathrm{C}$ for $11 \mathrm{~min}$. After such time, the crude reaction was purified by flash chromatography [hexane/EtOAc, 8:2] to afford the desired product as a solid. Table 2, entry 8. FTIR (neat): 3400 , 1579, 1301, 1149, $547 \mathrm{~cm}^{-1}$; mp 178-181 ${ }^{\circ} \mathrm{C}:{ }^{1} \mathrm{H}$ NMR $\left(400 \mathrm{MHz}, \mathrm{CDCl}_{3}\right) \delta 7.73(\mathrm{dd}, J=8.5,6.6$ $\mathrm{Hz}, 1 \mathrm{H}), 6.42$ (ddd, $J=13.5,9.7,2.0 \mathrm{~Hz}, 2 \mathrm{H}), 6.19(\mathrm{~s}, 1 \mathrm{H}), 5.98-5.83(\mathrm{~m}, 1 \mathrm{H}), 5.67$ (qt, $J=15.0$, $7.5 \mathrm{~Hz}, 1 \mathrm{H}), 5.25(\mathrm{dd}, J=19.5,13.8 \mathrm{~Hz}, 2 \mathrm{H}), 5.12(\mathrm{dd}, J=25.7,13.7 \mathrm{~Hz}, 2 \mathrm{H}), 4.67(\mathrm{t}, J=5.6 \mathrm{~Hz}$, $1 \mathrm{H}), 3.88-3.74(\mathrm{~m}, 2 \mathrm{H}), 3.52(\mathrm{t}, J=5.8 \mathrm{~Hz}, 2 \mathrm{H}) ;{ }^{13} \mathrm{C} \mathrm{NMR}\left(126 \mathrm{MHz}, \mathrm{CDCl}_{3}\right) \delta 167.8,165.8,147.9$ $\left(\mathrm{d}, J_{\mathrm{C}-\mathrm{F}}=13.1 \mathrm{~Hz}\right), 133.2,132.7,117.8,116.9,103.5,103.3,99.6,99.4,46.1$; HRMS calcd for $\mathrm{C}_{12} \mathrm{H}_{16} \mathrm{FN}_{2} \mathrm{O}_{2} \mathrm{~S}(\mathrm{M}+\mathrm{H})^{+} 271.0917$; found 271.0923 .

17. General procedure for the synthesis of benzothiadiazin-3-one-1,1-dioxides via CDI cyclization: To a round-bottomed flask was added sulfonamide ( $0.17 \mathrm{mmol}, 1$ equiv), dry DMF $(0.2 \mathrm{M}), \mathrm{Et}_{3} \mathrm{~N}(0.34$ mmol, 2 equiv) and CDI $\left(0.69 \mathrm{mmol}, 4\right.$ equiv). The reaction mixture was heated at $100{ }^{\circ} \mathrm{C}$ for $6 \mathrm{~h}$, cooled to rt and concentrated under reduced pressure. The crude oil was diluted in $\mathrm{CH}_{2} \mathrm{Cl}_{2}$, washed with $1 \mathrm{M} \mathrm{HCl}$ (aq, $5 \mathrm{~mL}$ ), water $(5 \mathrm{~mL})$, and dried $\left(\mathrm{MgSO}_{4}\right)$. Subsequent filtration and concentration yielded a crude oil which was purified by flash chromatography [hexane/EtOAc, 7:3] to afford the desired product as clear oil. (Table 3, entry 3). FTIR (neat): $3400,1575,1310,1149 \mathrm{~cm}^{-1}$; ${ }^{1} \mathrm{H}$ NMR $\left(500 \mathrm{MHz}, \mathrm{CDCl}_{3}\right) \delta 7.92-7.83(\mathrm{~m}, 1 \mathrm{H}), 7.03-6.94(\mathrm{~m}, 2 \mathrm{H}), 6.04-5.88(\mathrm{~m}, 2 \mathrm{H}), 5.39-5.30(\mathrm{~m}, 2 \mathrm{H})$, 5.29-5.19 (m, 2H), 4.69-4.59 (m, 2H), 4.52-4.46 (m, 2H); $\left.{ }^{13} \mathrm{C} \mathrm{NMR} \mathrm{(126} \mathrm{MHz,} \mathrm{CDCl}_{3}\right) \delta 166.7$, $164.6,150.2,138.8\left(\mathrm{dd}, J_{\mathrm{C}-\mathrm{F}}=10.8 \mathrm{~Hz}\right), 131.6,130.7,125.3,119.2,118.0,111.1,104.7,104.4$, 48.5, 44.8; HRMS calcd for $\mathrm{C}_{13} \mathrm{H}_{13} \mathrm{FN}_{2} \mathrm{O}_{2} \mathrm{~S}(\mathrm{M}+\mathrm{H})^{+}$297.0709; found 297.0712.

18. General one-pot procedure for the synthesis of benzothiadiazin-3-one-1,1-dioxides: Into a microwave reaction vial $(0.5-2.0 \mathrm{ml})$ was added sulfonamide ( $0.17 \mathrm{mmol}, 1$ equiv), $\mathrm{CuI}(0.017 \mathrm{mmol} .0 .1$ equiv), 1,10-phenanthroline ( $0.034 \mathrm{mmol}, 0.2$ equiv), $\mathrm{Cs}_{2} \mathrm{CO}_{3}$ ( $0.34 \mathrm{mmol}, 2$ equiv), dry solvent ( $\left.0.5 \mathrm{M}\right)$, and amine $(0.2 \mathrm{mmol}, 1.2$ equiv). The reaction was heated in the microwave (Biotage initiator, www.biotage.com) at $150{ }^{\circ} \mathrm{C}$ for $11 \mathrm{~min}$. After such time $\mathrm{Et}_{3} \mathrm{~N}(0.34 \mathrm{mmol}, 2$ equiv) and CDI (0.69 mmol, 4 equiv) was added directly to the microwave vial. The reaction mixture was heated at $150^{\circ}$ $\mathrm{C}$ for $11 \mathrm{~min}$, cooled to $\mathrm{rt}$ and concentrated under reduced pressure. The crude oil was diluted in $\mathrm{CH}_{2} \mathrm{Cl}_{2}$, washed with $1 \mathrm{M} \mathrm{HCl}(\mathrm{aq}, 5 \mathrm{~mL})$, water $(5 \mathrm{~mL})$ and dried $\left(\mathrm{MgSO}_{4}\right)$. Subsequent filtration and concentration yielded a crude oil, which was purified by flash chromatography [hexane/EtOAc, $7: 3$ ] to afford the desired product. 
<smiles>O=C1Nc2ccc(Cl)cc2S(=O)(=O)N1</smiles><smiles>O=C1Nc2ccccc2S(=O)(=O)N1</smiles>

Hypoglycemic agent

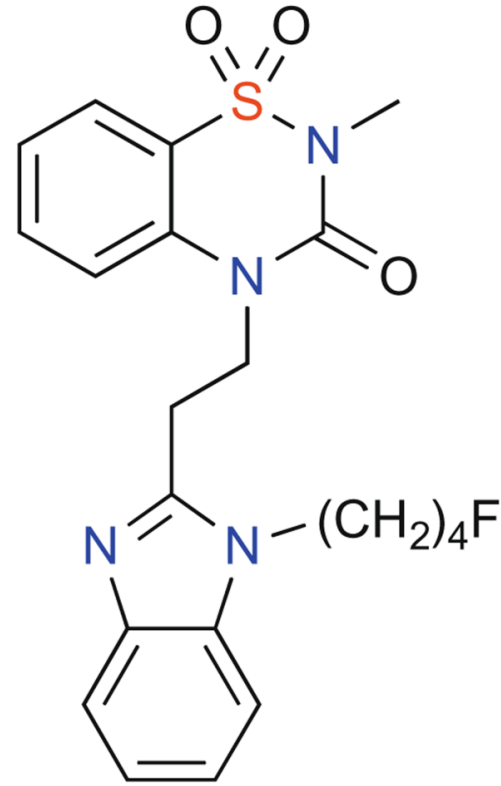

RSV Inhibitors<smiles>CN1C(=O)N(Br)S(=O)(=O)c2cscc21</smiles>
Non-Nucleoside reverse Transcriptase inhibitor

Figure 1.

Biologically active benzofuzed sultams. 

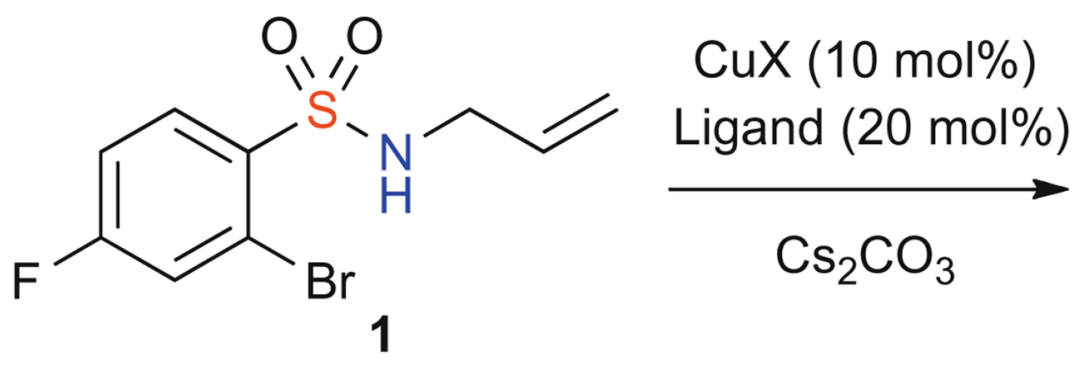

Scheme 1.

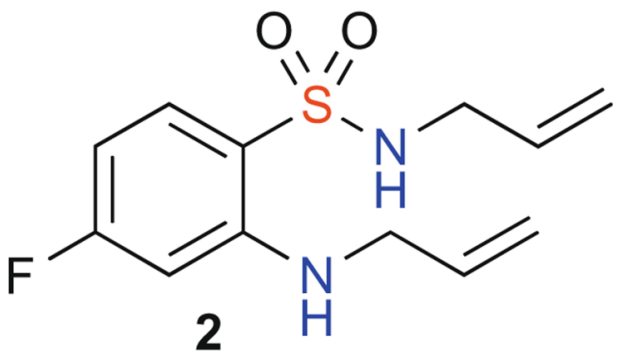


<smiles>[R]NS(=O)(=O)c1cc([R])c([R2])cc1Br</smiles>

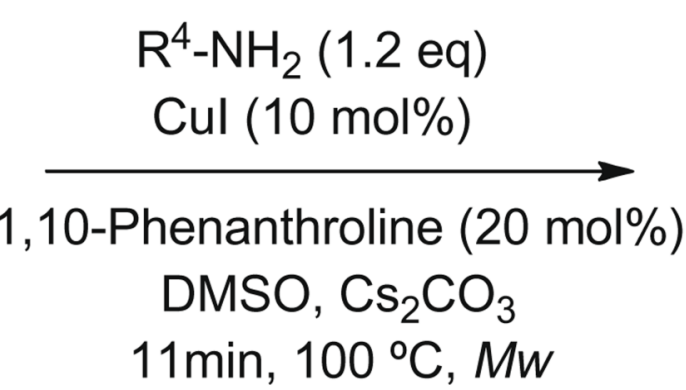<smiles>[R]Nc1cc([R])c([R])cc1S(=O)(=O)N[R]</smiles>

Scheme 2. 

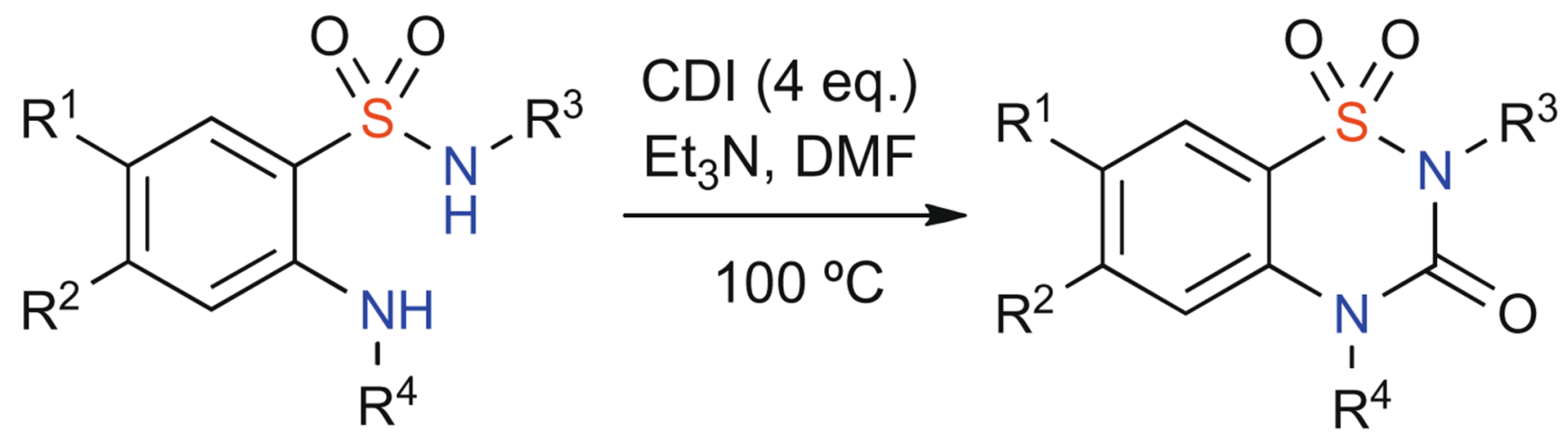

Scheme 3. 


$$
\begin{aligned}
& \mathrm{R}^{1}=\mathrm{H}, \mathrm{R}^{2}=\mathrm{Allyl}, \mathrm{R}^{3}=\mathrm{Allyl}, 72 \% \\
& \mathrm{R}^{1}=\mathrm{F}, \mathrm{R}^{2}=4-\mathrm{MeOBn}, \mathrm{R}^{3}=\mathrm{Octyl}, 68 \% \\
& \mathrm{R}^{1}=\mathrm{H}, \mathrm{R}^{2}=\mathrm{Allyl}, \mathrm{R}^{3}=\mathrm{OBn}, 73 \%
\end{aligned}
$$

Scheme 4. 
Table 1

Screening conditions for reaction optimization

\begin{tabular}{|c|c|c|c|c|}
\hline Entry $a, d$ & {$[\mathrm{Cu}]$ cat. } & Ligand & Solvent & Yield (\%) \\
\hline 1 & $\mathrm{CuI}$ & L-Proline & DMSO & 65 \\
\hline 2 & $\mathrm{CuBr}$ & L-Proline & DMSO & 55 \\
\hline 3 & $\mathrm{Cu}_{2} \mathrm{O}$ & L-Proline & DMSO & 10 \\
\hline 4 & $\mathrm{CuI}$ & $\left(\mathrm{CH}_{2} \mathrm{OH}\right)_{2}$ & DMSO & 78 \\
\hline 5 & $\mathrm{CuI}$ & 1,10-Phenanthroline & DMSO & 94 \\
\hline 6 & $\mathrm{CuI}$ & DBU & DMSO & 50 \\
\hline 7 & $\mathrm{CuI}$ & $\left(\mathrm{CH}_{2} \mathrm{NHMe}\right)_{2}$ & DMSO & 72 \\
\hline 8 & $\mathrm{CuI}$ & 1,10-Phenanthroline & DMF & 92 \\
\hline 9 & $\mathrm{CuI}$ & 1,10-Phenanthroline & Dioxane & 84 \\
\hline 10 & $\mathrm{CuI}$ & 1,10-Phenanthroline & DMF & $96^{b}$ \\
\hline 11 & $\mathrm{CuI}$ & 1,10-Phenanthroline & DMF & $94^{c}$ \\
\hline
\end{tabular}

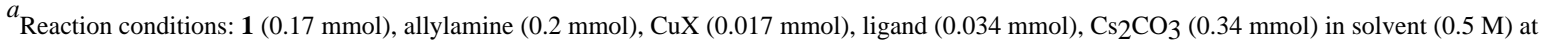
$100{ }^{\circ} \mathrm{C}$ for $6 \mathrm{~h}$.

${ }^{b}$ Microwave irradiation for $22 \mathrm{~min}$ at $140{ }^{\circ} \mathrm{C}$.

${ }^{c}$ Microwave irradiation for $11 \mathrm{~min}$ at $150{ }^{\circ} \mathrm{C}$.

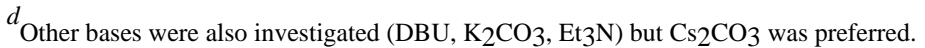




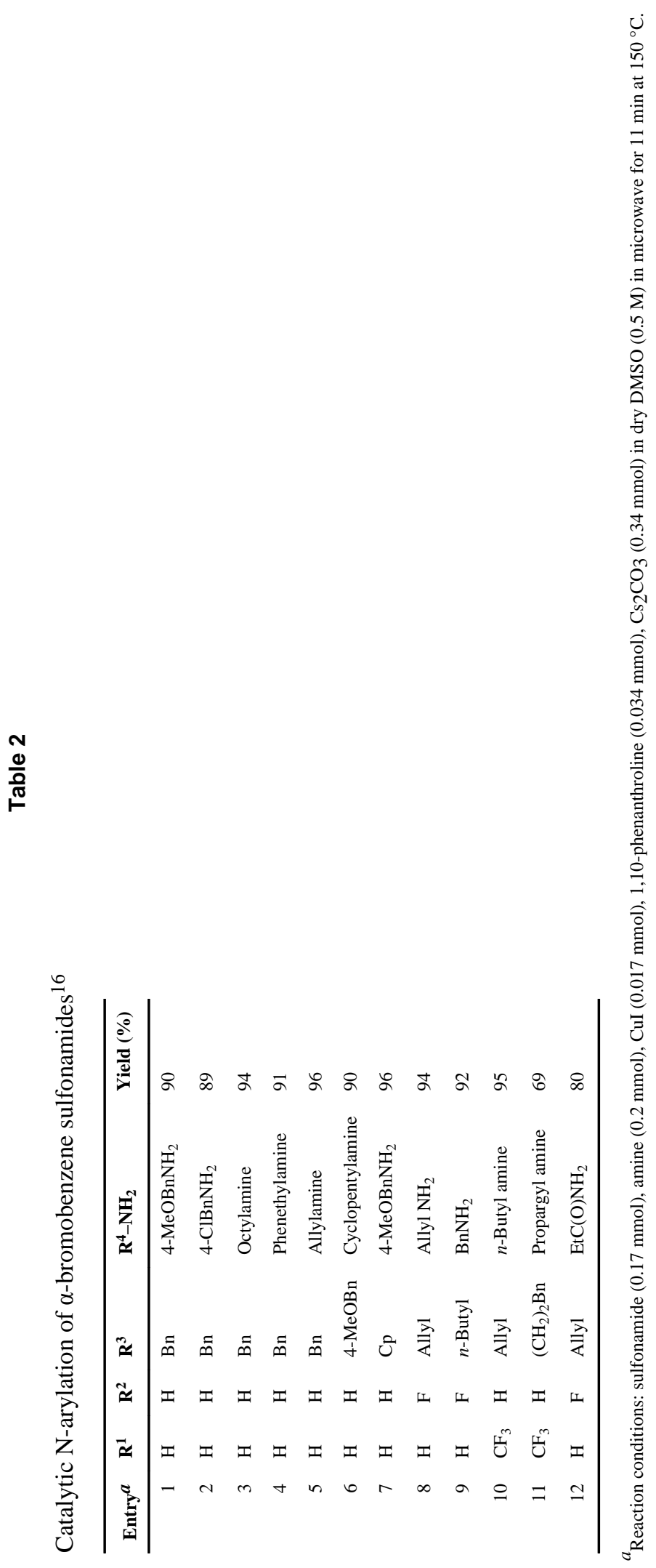

Tetrahedron Lett. Author manuscript; available in PMC 2010 December 1. 


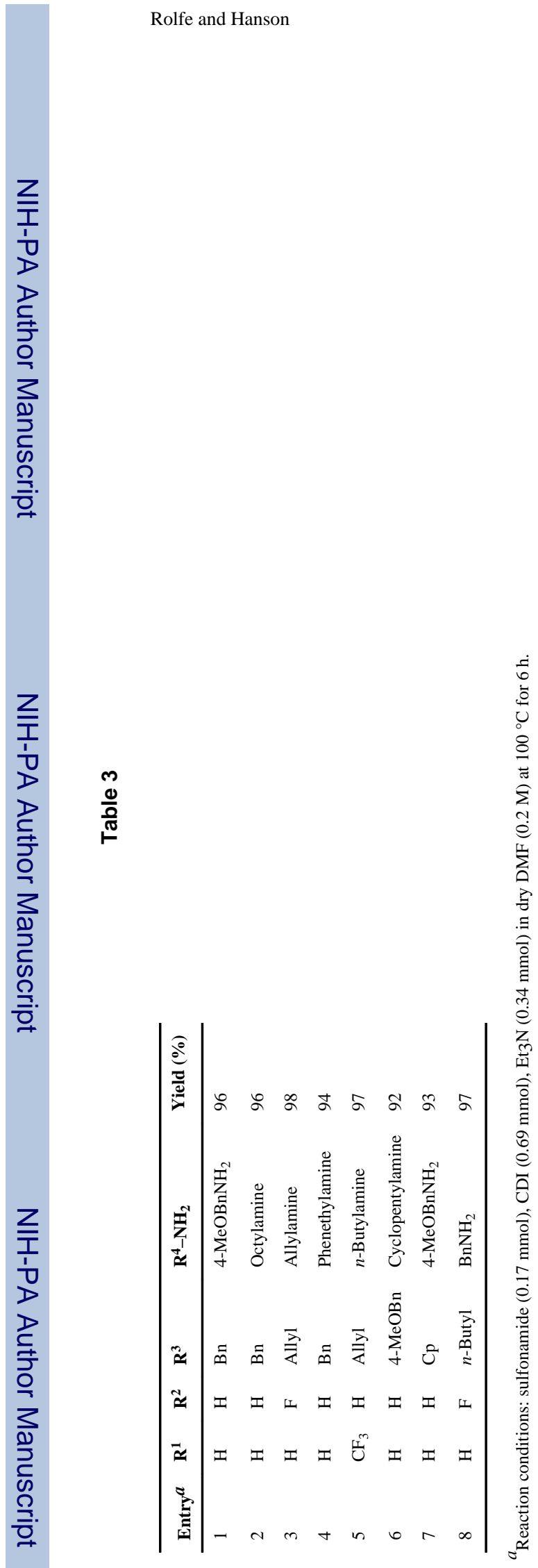

Tetrahedron Lett. Author manuscript; available in PMC 2010 December 1. 